\title{
TRAIL is involved in CpG ODN-mediated anti-apoptotic signals
}

\author{
EUN-JUNG LIM ${ }^{1 *}$, DAE-WEON PARK ${ }^{1 *}$, TAE-WHAL JEONG ${ }^{2}$, \\ BYUNG-RHO CHIN ${ }^{2}$, YOE-SIK BAE ${ }^{3}$ and SUK-HWAN BAEK ${ }^{1}$ \\ ${ }^{1}$ Department of Biochemistry and Molecular Biology, Aging-Associated Vascular Disease Research Center, \\ and ${ }^{2}$ Department of Dentistry, College of Medicine, Yeungnam University, Daegu; ${ }^{3}$ Department of \\ Biological Sciences, Sungkyunkwan University, Suwon, Republic of Korea
}

Received October 5, 2011; Accepted November 16, 2011

DOI: 10.3892/or.2011.1579

\begin{abstract}
Synthetic oligodeoxynucleotides (ODNs) with the CpG-motifs are recognized by toll-like receptor 9 (TLR9), which elicits an immune response. Serum starvation of Raw264.7 cells increased tumor necrosis factor-related apoptosis-inducing ligand (TRAIL) expression. However, treatment with $\mathrm{CpG}$ ODN reduced TRAIL expression as well as apoptosis by serum starvation. In serum starved cells, TLR9 inhibitors recovered the decreasing TRAIL expression and sub-G1 accumulation by CpG ODN. CpG ODN-regulated anti-apoptotic signals which were dependent on the Akt-FoxO3a signaling pathway. $\mathrm{CpG}$ ODNs activated Akt and inactivated FoxO3a in serum starved cells. Knockdown of FoxO3a by siRNA decreased TRAIL expression and apoptosis in serum-starved cells. In contrast, FoxO3a overexpression increased apoptosis by serum starvation, and $\mathrm{CpG}$ ODNs blocked these effects through TRAIL expression. LY294002, a PI3K-Akt inhibitor, blocked the CpG ODN effect of TRAIL expression and the sub-G1 population in serum starved cells. In contrast, overexpression of wild-type Akt reduced additional sub-G1 cells both in non-CpG ODNand $\mathrm{CpG}$ ODN-treated cells. Taken together, these results demonstrate the involvement of Akt-FoxO3a signaling in TLR9mediated downregulation of TRAIL and anti-apoptotic signals.
\end{abstract}

\section{Introduction}

Toll-like receptors (TLRs) recognize a set of conserved molecular structures called pathogen-associated molecular patterns,

Correspondence to: Dr Suk-Hwan Baek, Department of Biochemistry and Molecular Biology, Aging-Associated Vascular Disease Research Center, 210 Main Building, College of Medicine, Yeungnam University, 317-1 Daemyung-5 Dong, Namgu, Daegu 705-802, Republic of Korea

E mail: sbaek@med.yu.ac.kr

*Contributed equally

Abbreviations: TRAIL, tumor necrosis factor-related apoptosisinducing ligand; $\mathrm{CpG}$ ODN, $\mathrm{CpG}$ oligodeoxynucleotide; TLR9, toll-like receptor 9; FoxO, forkhead transcription factors of the $\mathrm{O}$ class

Key words: CpG ODN, FoxO3a, TRAIL, anti-apoptosis which allow them to sense innate and adaptive immune responses. Among them, TLR9 is essential for recognition of microbial CpG DNA or synthetic $\mathrm{CpG}$ oligonucleotide analogs containing a $\mathrm{CpG}$ oligodeoxynucleotide (ODN). CpG DNA activates macrophages, monocytes, and dendritic cells to secrete proinflammatory cytokines $(1,2)$. The binding of CpG DNA to TLR9 and subsequent endosomal maturation are thought to be essential for CpG DNA-driven immunostimulatory activity (3). After CpG DNA binding, TLR9 signaling is initiated by recruitment of the adaptor molecule MyD88 followed by the engagement of interleukin (IL)-1R-associated kinases and tumor necrosis factor (TNF)- $\alpha$ receptor (TNFR)-associated factor 6 (4). These complexes activate the IкB kinase complex and subsequently activate NF- $\mathrm{\kappa B}$-dependent pro-inflammatory cytokines such as TNF- $\alpha$ and IL-1 $\beta$ (5). TLRs are members of the IL-1R superfamily and share a common activation pathway through their Toll/IL-1R signaling domain (6). Despite this common pathway, TLRs show differences in their rate, intensity, or efficiency of activation by yet unidentified mechanisms.

Members of the FoxO subfamily of forkhead transcription factors include the mammalian ortholog DAF-16, which regulates longevity in the nematode Caenorhabditis elegans (7). Mice and humans possess three highly related FoxO homologs (FoxO1, FoxO3 and FoxO4) with overlapping expression patterns and transcriptional activities (8). Suppression of FoxO transcriptional activity by Akt-mediated phosphorylation leads to enhanced cell survival (9). In conditions in which the Akt survival and growth pathway is activated, FoxO3a is phosphorylated by Akt and exported to the cytoplasm (10). In contrast, unphosphorylated FoxO3a proteins are active forms and are located in the nucleus where they bind to their target gene promoters. Overexpression of the constitutively activated form of FoxO3a leads to apoptosis in many cell types (11). Additionally, FoxO3a mediates apoptosis by activating pro-apoptotic genes such as TNF-related apoptosis-inducing ligand (TRAIL) (12). Although FoxO3a has generally been considered an inducer of apoptosis, there is little evidence of TLR signaling.

In this study, we investigated the role of TRAIL in TLR9mediated anti-apoptosis of macrophages. We found that $\mathrm{CpG}$ ODN treatment blocked serum deprivation-mediated apoptosis. We also found that CpG ODN downregulated TRAIL gene expression. We further investigated the mechanisms of CpG ODN-induced TRAIL expression via the TLR9-AktFoxO3a signaling pathway. 
A

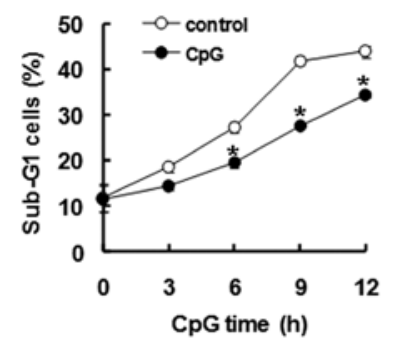

C

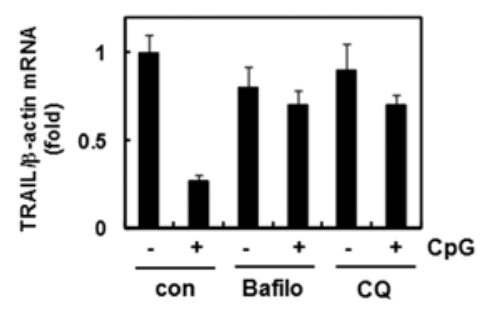

B

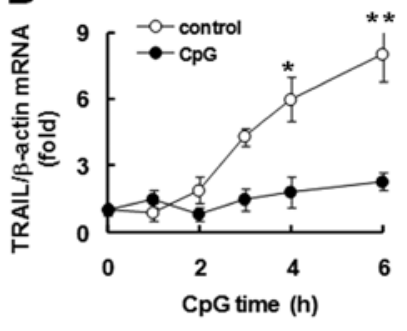

D

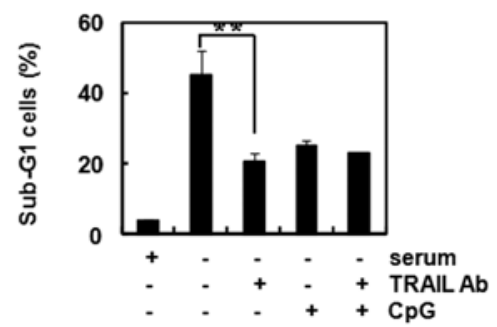

Figure 1. Synthetic oligodeoxynucleotides (ODN) with the CpG-motif (CpG ODN) inhibit apoptosis induced by serum starvation by downregulating tumor necrosis factor-related apoptosis-inducing ligand (TRAIL) in macrophages. (A) Raw264.7 cells were cultured, changed to serum-free medium, and incubated with medium (control) or $3 \mu \mathrm{M} \mathrm{CpG} \mathrm{ODN} \mathrm{(CpG)} \mathrm{for} \mathrm{the} \mathrm{indicated} \mathrm{times.} \mathrm{Cells} \mathrm{were} \mathrm{stained} \mathrm{with} \mathrm{propidium} \mathrm{iodide} \mathrm{(PI)} \mathrm{and} \mathrm{analyzed} \mathrm{for} \mathrm{apoptosis} \mathrm{by} \mathrm{flow}$ cytometry. (B) Cultured cells were changed to serum-free medium and incubated with $3 \mu \mathrm{M}$ CpG ODN for the indicated times. Total RNA was then isolated from the cells and subjected to real-time RT-PCR. The graph represents the fold-changes in TRAIL mRNA after the cells were treated with $3 \mu \mathrm{M} \mathrm{CpG} \mathrm{ODN.}$ Each data point represents the mean \pm SD of three independent experiments $\left({ }^{*} \mathrm{P}<0.05\right)$. (C) Cultured cells were changed to serum free medium and pretreated with $5 \mathrm{nM}$ bafilomycin A1 or $5 \mu \mathrm{g} / \mathrm{ml} \mathrm{CQ}$ prior to CpG ODN stimulation for $6 \mathrm{~h}$. mRNA levels were analyzed by real-time PCR using specific TRAIL or $\beta$-actin primers. (D) Cells were pretreated with neutralizing TRAIL antibody (Ab), CpG ODN, or a combination of TRAIL Ab plus CpG ODN in serum-free medium for $12 \mathrm{~h}$. The cells were stained with PI and analyzed for the induction of apoptosis by flow cytometry. Each data point represents the mean \pm SD of three independent experiments $\left({ }^{* *} \mathrm{P}<0.01\right)$.

\section{Materials and methods}

Reagents and antibodies. Cell culture reagents were obtained from Life Technologies (Grand Island, NY, USA). Fetal bovine serum (FBS) was obtained from Thermo Scientific HyClone (Logan, UT). Chloroquine, propidium iodide (PI) and $\beta$-actin antibody were obtained from Sigma-Aldrich (St. Louis, MO, USA). Phosphorothioated unmethylated endotoxin-free CpG ODN (B-class, TCCATGACGTTCCTGATGCT) and control ODN 1720 (TCCATGAGCTTCCTGATGCT, inactive control for CpG ODN 1668) were obtained from Genotech (Daejeon, South Korea), and an RNA reverse transcriptionpolymerase chain reaction (RT-PCR) core kit was purchased from Axygen Biosciences (Union City, CA, USA). Antibodies (Abs) against FoxO3a and Akt were purchased from Cell Signaling Technology (Beverly, MA, USA). Bafilomycin A1 and LY294002 were purchased from Calbiochem (San Diego, CA, USA).

Cell culture. The Raw264.7 macrophage cell line was obtained from the American Type Culture Collection (Manassas, VA, USA). Cells were grown in Dulbecco's modified Eagle's medium (Invitrogen, Carlsbad, CA, USA) containing 10\% FBS, $2 \mu \mathrm{M}$ L-glutamine, $10 \mathrm{U} / \mathrm{ml}$ penicillin and $10 \mu \mathrm{g} / \mathrm{ml}$ streptomycin at $37^{\circ} \mathrm{C}$ in a humidified atmosphere under $5 \%$ $\mathrm{CO}_{2}$. Cells were treated with synthetic $\mathrm{CpG}$ ODN for various times.

Fluorescence-activated cell sorting (FACS) analysis. To quantify apoptotic nuclei, cells were fixed in ethanol, stained with $50 \mu \mathrm{g} / \mathrm{ml}$ PI and RNase A for $30 \mathrm{~min}$ at room temperature followed by washing, and the samples were processed by flow cytometry using a FACSCalibur apparatus (BD Biosciences, Franklin Lakes, NJ, USA). The results are shown as a histogram with sub-G1 positive cells considered the apoptotic cells.

Western blot analysis. The cells were washed with cold-PBS, trypsinized, and pelleted at $700 \mathrm{x}$ g. Cell pellets were resuspended in lysis buffer comprised of $50 \mathrm{mM}$ Tris- $\mathrm{HCl}$ ( $\mathrm{pH} \mathrm{8.0)}$, $5 \mathrm{mM}$ EDTA, $150 \mathrm{mM} \mathrm{NaCl}$, 0.5\% Nonidet P-40, $1 \mathrm{mM}$ PMSF, and a protease inhibitor cocktail. The preparations were then cleared by centrifugation, and the supernatants were saved as cell lysates. Proteins were separated by $8 \%$ reducing sodium dodecyl sulfate-polyacrylamide gel electrophoresis and immunoblotted in $20 \%$ methanol, $25 \mathrm{mM}$ Tris, and $192 \mathrm{mM}$ glycine onto nitrocellulose membranes. The membranes were then blocked with $5 \%$ non-fat dry milk in TTBS $(25 \mathrm{mM}$ Tris- $\mathrm{HCl}, 150 \mathrm{mM} \mathrm{NaCl}$, and $0.2 \%$ Tween-20) and incubated with primary $\mathrm{Ab}$ for $4 \mathrm{~h}$. Subsequently, membranes were washed, incubated for $1 \mathrm{~h}$ with secondary $\mathrm{Ab}$ conjugated to horseradish peroxidase, rewashed, and finally developed using an enhanced chemiluminescence system (Amersham, Buckinghamshire, UK).

Real-time RT-PCR. Total RNA was extracted from cells using TRIzol reagent (Invitrogen). Total RNA ( $1 \mu \mathrm{g})$ was used as a template to make first strand cDNA by oligo-dT priming using a reverse transcriptase system (Promega, Madison, WI, USA). Real-time RT-PCR was performed using a LightCycler 1.5 
(Roche Diagnostics, Almere, The Netherlands) with SYBRGreen I as the florescent dye, according to the manufacturer's instructions. The synthetic gene-specific primer sets used for PCR were: i) TRAIL forward primer, 5'-CCTCTCGGAAAG GGCATT C-3', and reverse primer, 5'-TCCTGCTCGATG ACCAGC T-3', which amplified $70 \mathrm{bp}$ of the mouse TRAIL cDNA; ii) $\beta$-actin forward primer, 5'-AGAGGGAAATCG TGCGTGAC-3', and reverse primer, 5'-CAATAGTGATGA CCTGGCCGT-3', which amplified 137 bp of the mouse $\beta$-actin cDNA. Cycling conditions were $95^{\circ} \mathrm{C}$ for $10 \mathrm{~min}$, followed by 45 cycles of $95^{\circ} \mathrm{C}$ for $10 \mathrm{sec}, 62^{\circ} \mathrm{C}$ for $5 \mathrm{sec}$, and $72^{\circ} \mathrm{C}$ for $6 \mathrm{sec}$. Target genes were normalized to $\beta$-actin for quantification.

Knock-down of FoxO3a using small interfering RNA (siRNA). Oligonucleotides corresponding to the mouse FoxO3a siRNA sequence 5'-UGAUGAUCCACCAAGAGCUCUUGCC-3' were purchased from Invitrogen. A control siRNA was also purchased and used. For transfection, $2 \times 10^{6}$ Raw 264.7 cells were resuspended in a nucleoporator buffer (Lonza, Allendale, NJ, USA) with 200 pmole siRNA. Cells were nucleoporated according to the manufacturer's protocol, and the above genes were knocked down for $24 \mathrm{~h}$.

FoxO3a and Akt overexpression. A vector encoding a FoxO3a protein (pLenti6/V5-D-TOPO-FoxO3a) was generously provided by Dr Kim (Yeungnam University, South Korea) for cell protein expression. Akt was subcloned into the pEGFP-C1 mammalian expression vector. Cells were transfected with the control vector, wild-type FoxO3a, or Akt cDNA for $24 \mathrm{~h}$, and fresh medium was added. Cells transfected with the cDNA were cloned by serial dilution in a 96-well plate in a culture medium with selecting antibiotics to obtain a stable cell line. Sub-culturing was continued for 4 weeks, and then wells representing a single colony were selected and expression was confirmed using the protein level as determined by Western blot analysis.

\section{Results}

CpG ODN blocks TRAIL expression and apoptosis using serum starvation. We first investigated the effect of CpG ODN in serum starvation-induced apoptosis. As expected, CpG ODN had a significant inhibitory effect on sub-G1 cell accumulation following serum starvation (Fig. 1A). The change of TRAIL mRNA expression using serum starvation in Raw264.7 cells was further examined. While serum starvation induced a dramatic increase in TRAIL expression in a time-dependent manner, CpG ODN strongly inhibited this response (Fig. 1B). We studied whether TLR9 mediates the inhibition of TRAIL expression and apoptosis. Pretreatment with bafilomycin A1 or chloroquine recovered TRAIL expression reduced by $\mathrm{CpG}$ ODN (Fig. 1C). To confirm the role of TRAIL in apoptosis following serum starvation, we treated Raw264.7 cells with the neutralizing TRAIL antibody or a combination with CpG ODN. Apoptosis partially but significantly decreased in the TRAIL neutralizing antibody-treated cells, and this effect was very similar to that of the CpG ODN treated cells. However, no additional effect was observed with the combination of $\mathrm{CpG}$ ODN and the TRAIL neutralizing antibody (Fig. 1D). These results suggest that $\mathrm{CpG}$ ODN plays a role
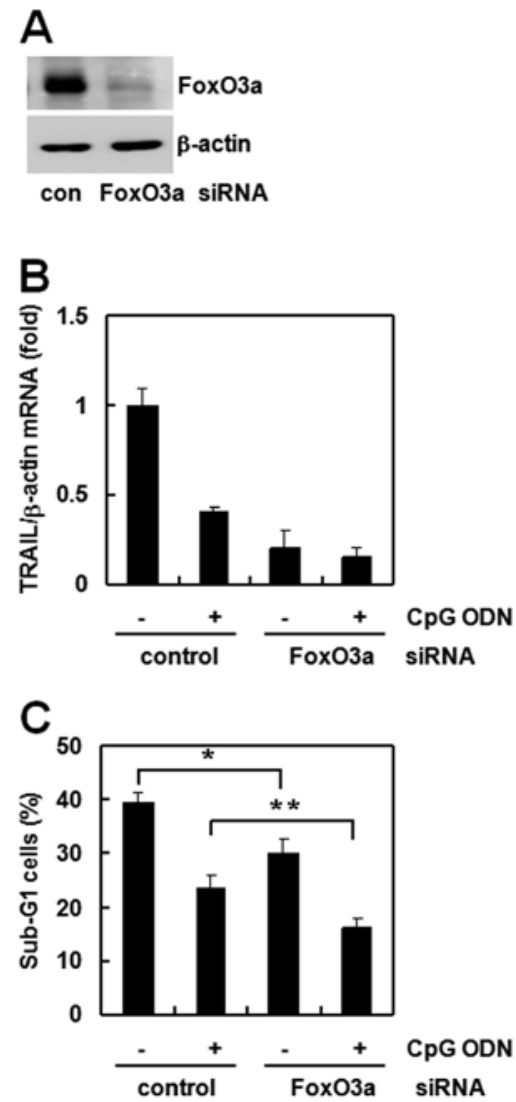

Figure 2. FoxO3a siRNA decreases tumor necrosis factor-related apoptosisinducing ligand (TRAIL) expression. Cells were transfected with control siRNA or FoxO3a siRNA. (A) FoxO3a expression was assessed by Western blotting using anti-FoxO3a antibody. (B) Control or FoxO3a siRNA cells were changed to serum free medium. After $9 \mathrm{~h}$, the cells were incubated with synthetic oligodeoxynucleotides (ODN) with the CpG-motif (CpG ODN) for $6 \mathrm{~h}$, and TRAIL mRNA levels were assessed by real-time PCR. (C) siRNA cells were changed to serum-free media and treated with $3 \mu \mathrm{M}$ CpG ODN for $12 \mathrm{~h}$, stained with propidium iodide (PI), and analyzed for the induction of apoptosis by flow cytometry. Each data point represents the mean \pm SD of four independent experiments $\left({ }^{*} \mathrm{P}<0.05\right.$ and $\left.{ }^{* *} \mathrm{P}<0.01\right)$.

in serum starvation-induced apoptosis by inhibiting TRAIL expression.

FoxO3a is involved in $C p G$ ODN-regulated TRAIL expression. FoxO3a is a well known transcription factor for TRAIL regulation (13). Therefore, we assessed the involvement of FoxO3a in $\mathrm{CpG}$ ODN-mediated TRAIL downregulation using siRNA or an overexpression technique. Transfection of FoxO3a siRNA effectively decreased protein expression in Raw264.7 cells (Fig. 2A). Then, we compared the $\mathrm{CpG}$ ODN effect between control and FoxO3a siRNA cells. TRAIL expression was reduced dramatically in FoxO3a siRNA cells compared to that of control siRNA cells. Treatment with $\mathrm{CpG}$ ODN reduced TRAIL expression (Fig. 2B) and sub-G1 accumulation in both siRNA cells (Fig. 2C). To confirm the role of FoxO3a, we overexpressed the gene in the same cell lines and investigated the effect of CpG ODN. TRAIL expression increased strongly in FoxO3a overexpressed cells compared to that of empty-vector transfected cells (Fig. 3A). However, $\mathrm{CpG}$ ODN treatment decreased TRAIL expression (Fig. 3B) and sub-G1 accumulation (Fig. 3C) in both types of transfected 


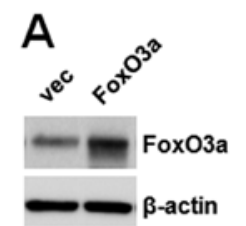

B
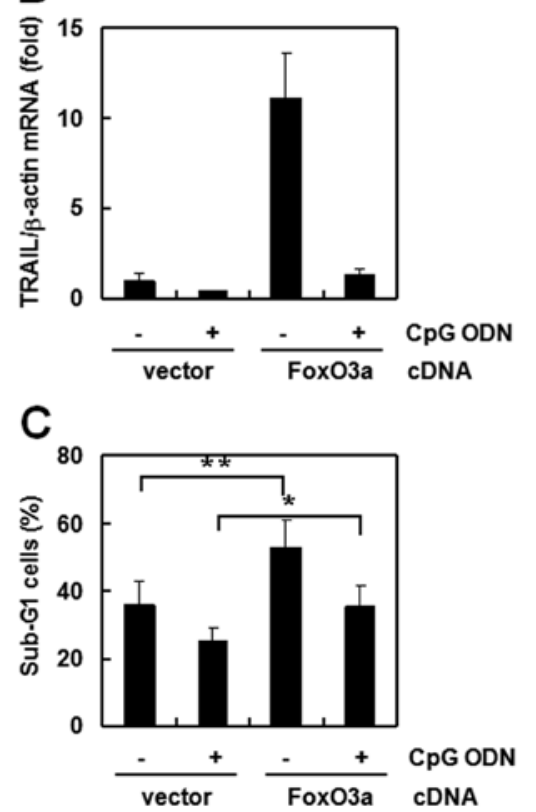

Figure 3. FoxO3a overexpression increases tumor necrosis factor-related apoptosis-inducing ligand (TRAIL) expression. Cells were stably transfected with a control vector (pLenti/V5-D-TOPO) or cDNA encoding wild-type FoxO3a. (A) FoxO3a expression was assessed by Western blotting using antiFoxO3a Ab. (B) Control vector or FoxO3a overexpressing cells were changed to serum-free medium. After $9 \mathrm{~h}$, cells were incubated with synthetic oligodeoxynucleotides (ODN) with the CpG-motif (CpG ODN) for $6 \mathrm{~h}$, and TRAIL mRNA levels were assessed by real-time PCR. (C) Vector or FoxO3a overexpressing cells were changed to serum free media and treated with $3 \mu \mathrm{M} \mathrm{CpG} \mathrm{ODN}$ for $12 \mathrm{~h}$, stained with propidium iodide (PI), and analyzed for the induction of apoptosis by flow cytometry. Each data point represents the mean $\pm \mathrm{SD}$ of three independent experiments $\left({ }^{*} \mathrm{P}<0.05\right.$ and $\left.{ }^{* *} \mathrm{P}<0.01\right)$.

cells. These results suggest that FoxO3a directly regulates TRAIL expression and that the anti-apoptosis effect of $\mathrm{CpG}$ ODN occurs through TRAIL.

The Akt-FoxO3a pathway is involved in regulating TRAIL expression and apoptosis. Akt is a well known apoptosis regulatory kinase, and $\mathrm{FoxO} 3$ a phosphorylation by Akt leads to inactivation of its transcriptional activity (14). Previously, we observed that $\mathrm{CpG}$ ODN phosphorylates Akt for activation and phosphorylates FoxO3a for inactivation (15). Therefore, we investigated the role of Akt in TRAIL expression and apoptosis by CpG ODN. While CpG ODN blocked serum starvation-induced TRAIL expression, LY294002 pretreatment partially and significantly recovered the reduced TRAIL expression (Fig. 4A). Apoptosis increased following LY294002 treatment (Fig. 4B). To confirm the role of Akt in apoptosis, we transfected the GFP vector or GFP-tagged Akt cDNA and compared the effect of CpG ODN. Akt expression increased in Akt cDNA transfected cells. Akt transfection itself decreased the sub-G1 population compared to that of vector-transfected cells, and the decrease was deepened by CpG ODN (Fig. 4C).
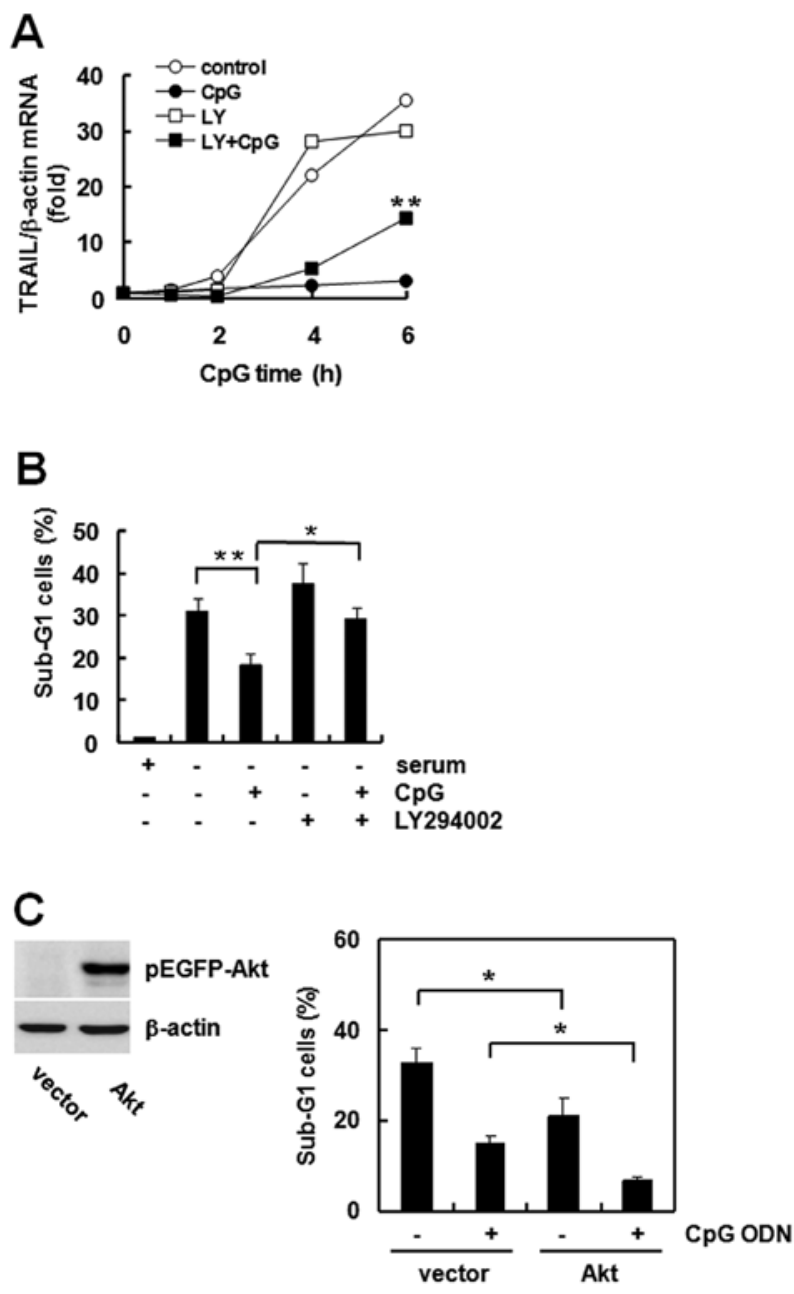

Figure 4. The Akt pathway is involved in synthetic oligodeoxynucleotides (ODN) with the CpG-motif (CpG ODN)-mediated anti-apoptosis through tumor necrosis factor-related apoptosis-inducing ligand (TRAIL) expression. (A) Cells were serum starved for $12 \mathrm{~h}$, pretreated with $10 \mu 1 \mathrm{LY} 294002$, and incubated with $3 \mu \mathrm{M} \mathrm{CpG} \mathrm{ODN} \mathrm{for} \mathrm{the} \mathrm{indicated} \mathrm{times.} \mathrm{TRAIL} \mathrm{mRNA}$ levels were assessed by real-time PCR. (B) Cells were changed to serum free medium, pretreated with $10 \mu \mathrm{M} \mathrm{LY} 294002$, and incubated with $3 \mu \mathrm{M} \mathrm{CpG}$ ODN for $12 \mathrm{~h}$. Cells were stained with propidium iodide (PI), and analyzed for the induction of apoptosis by flow cytometry. Each data point represents the mean \pm SD of four independent experiments $\left({ }^{*} \mathrm{P}<0.05\right.$ and $\left.{ }^{* *} \mathrm{P}<0.01\right)$. (C) Cells were transiently transfected with a control vector (pEGFP) or cDNA encoding wild-type Akt. Akt expression was assessed by Western blotting using anti-Akt antibody. Vector or Akt overexpressing cells were changed to serum-free medium and treated with $3 \mu \mathrm{M} \mathrm{CpG} \mathrm{ODN} \mathrm{for} 12 \mathrm{~h}$, stained with PI, and analyzed for the induction of apoptosis by flow cytometry. Each data point represents the mean $\pm \mathrm{SD}$ of four independent experiments $\left({ }^{*} \mathrm{P}<0.05\right)$.

These results suggest that Akt is a very important regulator of CpG ODN-mediated TRAIL expression and anti-apoptosis.

\section{Discussion}

TLRs including TLR9 play a central role in innate immunity by mediating pathogen recognition (16). Previous studies have demonstrated a role for TLR9 in mediating the effects of cell survival including macrophages. We also observed that treating macrophages with TLR9 agonists strongly obviated apoptosis. Many groups have investigated the regulatory proteins involved in cell survival, but the specific proteins involved in the response to a TLR9 agonist have not been clarified. CpG ODN promotes 
cell survival via Hsp70 upregulation to increase Bcl-xL (17). Furthermore, Hsp90 $\beta$ is also involved in the TLR9 anti-apoptotic effect (18). Therefore, we examined the effect of a TLR9 agonist on the cellular levels of the many proteins participating in apoptotic pathways. Among the possible regulatory factors, our results identified TRAIL as an important regulator of macrophages in apoptosis.

Our results demonstrate that $\mathrm{CpG}$ ODN strongly downregulated TRAIL expression in macrophages via the TLR9-dependent pathway. TRAIL is involved in apoptosis signaling pathways, specifically by modulating immune system function (19). Interestingly, TRAIL receptor-mediated apoptosis is inhibited by FLIP, through suppression of either recruitment of procaspase- 8 by FADD or autocatalytic activation of caspase-8 $(20,21)$. Therefore, our results suggest that TRAIL is suppressed after $\mathrm{CpG}$ ODN treatment, and that these responses could interfere with serum deprivation-induced apoptosis.

$\mathrm{NF}-\kappa \mathrm{B}$ is a transcription factor that potentially affects the expression of many genes and may favor cell survival by upregulating gene products with anti-apoptotic properties or downregulating pro-apoptotic factors (22). It has been suggested that activating NF- $\kappa \mathrm{B}$ via the TLR2 signaling pathway and the subsequent induction of gene expression can protect cells from FasL-induced apoptosis (23). Recognition of TLR9 by CpG ODN is also followed by NF- $\kappa \mathrm{B}$ activation (3). Despite this, the expression of several apoptosis-regulating genes is controlled by other transcription factors including FoxO (24).

Previous studies have suggested that activating Akt negatively regulates FoxO transcription factors $(9,25)$, and that the direct phosphorylation of Akt inhibits FoxO3a transcriptional activation (26). Additionally, the PI3K/Akt pathway is very important for the anti-apoptosis effects of CpG ODN (27). Furthermore, because Akt activity prevents the induction of apoptosis by cytokines, growth factors, and cellular stress (28), we determined the effect of the Akt pathway on FoxO3a activation, TRAIL expression, and apoptosis. Use of the pharmacological inhibitor LY294002 enabled us to assess the role of the Akt signaling pathway in the regulation of FoxO3a phosphorylation. Our data show that inhibiting Akt resulted in a significant increase in TRAIL gene expression in these cells. Furthermore, direct evidence was obtained by wild-type FoxO3a overexpression or using siRNA. Our data demonstrate that $\mathrm{CpG}$ ODN treatment increased TRAIL expression in FoxO3a overexpressing cells compared to vector control cells. FoxO3a siRNA cells demonstrated an opposite result compared to that of overexpressing cells. Together, these data strongly support the conclusion that FoxO3a is a transcription factor for TRAIL regulation in response to CpG ODN.

CpG ODN protects B-cells and macrophages against apoptosis $(17,29)$. Based on this result, we confirmed that engaging TLR9 protected against serum deprivation-induced apoptosis. Furthermore, we showed that $\mathrm{CpG}$ ODN induced an increase in FoxO3a phosphorylation. This protective effect was controlled by decreased TRAIL expression in $\mathrm{CpG}$ ODN-stimulated macrophages. The anti-apoptotic effects of CpG ODN stimulation require participation of the Akt-FoxO3a signaling pathway. Taken together, these results suggest that TLR9 triggers the FoxO3a transcription factor through Akt signaling, and that the regulation of TRAIL by CpG ODN may contribute to the anti-apoptotic effect.

\section{Acknowledgements}

This research was supported by Yeungnam University research grants in 2010 (210-A-356-007).

\section{References}

1. Ivanov S, Dragoi AM, Wang X, et al: A novel role for HMGB1 in TLR9-mediated inflammatory responses to CpG-DNA. Blood 110: 1970-1981, 2007.

2. Sanjuan MA, Rao N, Lai KT, et al: CpG-induced tyrosine phosphorylation occurs via a TLR9-independent mechanism and is required for cytokine secretion. J Cell Biol 172: 1057-1068, 2006.

3. Takeshita F, Gursel I, Ishii KJ, Suzuki K, Gursel M and Klinman DM: Signal transduction pathways mediated by the interaction of $\mathrm{CpG}$ DNA with Toll-like receptor 9. Semin Immunol 16: 17-22, 2004

4. Kawai T, Sato S, Ishii KJ, et al: Interferon-alpha induction through Toll-like receptors involves a direct interaction of IRF7 with MyD88 and TRAF6. Nat Immunol 5: 1061-1068, 2004.

5. Bagchi A, Herrup EA, Warren HS, et al: MyD88-dependent and MyD88-independent pathways in synergy, priming, and tolerance between TLR agonists. J Immunol 178: 1164-1171, 2007.

6. Bulek K, Swaidani S, Qin J, et al: The essential role of single Ig IL-1 receptor-related molecule/Toll IL-1R8 in regulation of Th2 immune response. J Immunol 182: 2601-2609, 2009.

7. Lee SS, Kennedy S, Tolonen AC and Ruvkun G: DAF-16 target genes that control $C$. elegans life-span and metabolism. Science 300: 644-647, 2003.

8. Paik JH, Kollipara R, Chu G, et al: FoxOs are lineage-restricted redundant tumor suppressors and regulate endothelial cell homeostasis. Cell 128: 309-323, 2007.

9. Zhao Y, Wang Y and Zhu WG: Applications of post-translational modifications of FoxO family proteins in biological functions. J Mol Cell Biol 3: 276-282, 2011.

10. Barthelemy C, Henderson CE and Pettmann B: Foxo3a induces motoneuron death through the Fas pathway in cooperation with JNK. BMC Neurosci 5: 48, 2004.

11. Cui M, Huang Y, Zhao Y and Zheng J: Transcription factor FOXO3a mediates apoptosis in HIV-1-infected macrophages. J Immunol 180: 898-906, 2008.

12. Modur V, Nagarajan R, Evers BM and Milbrandt J: FOXO proteins regulate tumor necrosis factor-related apoptosis inducing ligand expression. Implications for PTEN mutation in prostate cancer. J Biol Chem 277: 47928-47937, 2002.

13. Sakoe Y, Sakoe K, Kirito K, Ozawa K and Komatsu N: FOXO3A as a key molecule for all-trans retinoic acid-induced granulocytic differentiation and apoptosis in acute promyelocytic leukemia. Blood 115: 3787-3795, 2010.

14. Guan H, Song L, Cai J, et al: Sphingosine kinase 1 regulates the Akt/FOXO3a/Bim pathway and contributes to apoptosis resistance in glioma cells. PLoS One 6: e19946, 2011.

15. Lim EJ, Park DW, Lee JG, et al: Toll-like receptor 9-mediated inhibition of apoptosis occurs through suppression of FoxO3a activity and induction of FLIP expression. Exp Mol Med 42: 712-720, 2010.

16. Takeda $\mathrm{K}$ and Akira $\mathrm{S}$ : Toll-like receptors in innate immunity. Int Immunol 17: 1-14, 2005.

17. Kuo CC, Liang SM and Liang CM: CpG-B oligodeoxynucleotide promotes cell survival via up-regulation of Hsp70 to increase Bcl-xL and to decrease apoptosis-inducing factor translocation. J Biol Chem 281: 38200-38207, 2006.

18. Kuo CC, Liang CM, Lai CY and Liang SM: Involvement of heat shock protein (Hsp)90 beta but not Hsp90 alpha in antiapoptotic effect of CpG-B oligodeoxynucleotide. J Immunol 178: 6100-6108, 2007.

19. Wang S and El-Deiry WS: TRAIL and apoptosis induction by TNF-family death receptors. Oncogene 22: 8628-8633, 2003.

20. Mitsiades N, Mitsiades CS, Poulaki V, Anderson KC and Treon SP: Intracellular regulation of tumor necrosis factorrelated apoptosis-inducing ligand-induced apoptosis in human multiple myeloma cells. Blood 99: 2162-2171, 2002.

21. Xiao CW, Asselin E and Tsang BK: Nuclear factor kappaBmediated induction of Flice-like inhibitory protein prevents tumor necrosis factor alpha-induced apoptosis in rat granulosa cells. Biol Reprod 67: 436-441, 2002. 
22. Kerbauy DM, Lesnikov V, Abbasi N, Seal S, Scott B and Deeg HJ: NF-kappaB and FLIP in arsenic trioxide (ATO)induced apoptosis in myelodysplastic syndromes (MDSs). Blood 106: 3917-3925, 2005

23. Loeuillet C, Martinon F, Perez C, Munoz M, Thome M and Meylan PR: Mycobacterium tuberculosis subverts innate immunity to evade specific effectors. J Immunol 177: 6245-6255, 2006.

24. Fu $\mathrm{Z}$ and Tindall DJ: FOXOs, cancer and regulation of apoptosis. Oncogene 27: 2312-2319, 2008.

25. Greer EL and Brunet A: FOXO transcription factors at the interface between longevity and tumor suppression. Oncogene 24: 7410-7425, 2005.

26. Khatri S, Yepiskoposyan H, Gallo CA, Tandon P and Plas DR: FOXO3a regulates glycolysis via transcriptional control of tumor suppressor TSC1. J Biol Chem 285: 15960-15965, 2010.
27. Dragoi AM, Fu X, Ivanov S, et al: DNA-PKcs, but not TLR9, is required for activation of Akt by CpG-DNA. EMBO J 24: 779-789, 2005

28. Francois S, El Benna J, Dang PM, Pedruzzi E, GougerotPocidalo MA and Elbim C: Inhibition of neutrophil apoptosis by TLR agonists in whole blood: involvement of the phosphoinositide 3-kinase/Akt and NF-kappaB signaling pathways, leading to increased levels of Mcl-1, A1, and phosphorylated Bad. J Immunol 174: 3633-3642, 2005.

29. Krieg AM: CpG motifs in bacterial DNA and their immune effects. Annu Rev Immunol 20: 709-760, 2002. 